\title{
A Global Solution of the Einstein-Maxwell Field Equations for Rotating Charged Matter
}

\author{
Andreas Georgiou \\ School of Physics Astronomy and Mathematics, University of Hertfordshire, Hatfield, UK \\ Email: a.georgiou@herts.ac.uk
}

Received June 27, 2012; revised July 31, 2012; accepted August 9, 2012

\begin{abstract}
A stationary axially symmetric exterior electrovacuum solution of the Einstein-Maxwell field equations was obtained. An interior solution for rotating charged dust with vanishing Lorentz force was also obtained. The two spacetimes are separated by a boundary which is a surface layer with surface stress-energy tensor and surface electric 4-current. The layer is the spherical surface bounding the charged matter. It was further shown, that all the exterior physical quantities vanished at the asymptotic spatial infinity where spacetime was shown to be flat. There are two different sets of junction conditions: the electromagnetic junction conditions, which were expressed in the traditional 3-dimensional form of classical electromagnetic theory; and the considerably more complicated gravitational junction conditions. It was shown that both - the electromagnetic and gravitational junction conditions-were satisfied. The mass, charge and angular momentum were determined from the metric. Exact analytical formulae for the dipole moment and gyromagnetic ratio were also derived. The conditions, under which the latter formulae gave Blackett's empirical result for rotating stars, were investigated.
\end{abstract}

Keywords: Gravitation; Exact Solutions; Einstein-Maxwell Equations; Rotation; Charged Dust

\section{Introduction}

There are difficulties in finding exact solutions of the Einstein or of the Einstein-Maxwell field equations for a volume distribution of rotating bounded matter [1]. Such solutions should consist of an interior filled with matter and an asymptotically flat vacuum or electrovacuum exterior, these being separated by a surface on which appropriate boundary conditions should be satisfied. The main aim of this work is to obtain an exterior and matching interior solution of the Einstein-Maxwell field equations with finite bounded rotating charged matter as a source of the spacetime. Due to the rotation, the boundary will actually be an oblate spheroid, but it is assumed that it is a spherical surface with equation $r=a$. The main objective and emphasis after all, is to see how far the attempt at finding a solution can be taken - a solution with finite bounded rotating matter as a source of the spacetime. The additional complication of spheroidal coor-dinates is avoided, in a problem which is already enormously complicated.

Most of the equations and expressions for the various physical quantities are difficult to derive and they require involved and lengthy analysis. It is not therefore possible or desirable to include these calculations in the paper, but directions in which to proceed are indicated.

\section{The Einstein-Maxwell Field Equations}

Consider electrically charged pressure-free matter (charged dust) bounded by the hypersurface $r=a$ and rotating with constant angular velocity about the polar axis $\theta=0$ under zero Lorentz force. It is assumed that the current is carried by the dust. The transformed expression (2.1) in [2] for the Weyl-Lewis-Papapetrou metric for a stationary axially symmetric spacetime $V$ is

$$
\begin{aligned}
& \mathrm{d} s^{2}=-e^{\mu}\left(\mathrm{d} r^{2}+r^{2} \mathrm{~d} \theta^{2}\right) \\
& -F^{-1}\left(r^{2} \sin ^{2} \theta-K^{2}\right) \mathrm{d} \phi^{2}-2 K \mathrm{~d} \phi \mathrm{d} t+F \mathrm{~d} t^{2}
\end{aligned}
$$

where we have taken the signature of the spacetime metric tensor $g_{\lambda \mu}$ to be -2 . It is implicit in the form (1) of the metric that we have assumed, without loss of generality, that $L F+K^{2}=r^{2} \sin ^{2} \theta$ and so the component $g_{33}$ of $g_{\lambda \mu}$ is $g_{33}=-L$. We shall use units $c=G=1$ where $c$ is the vacuum speed of light and $G$ the Newtonian gravitational constant. Unless otherwise specified, we shall adopt the convention in which Roman indices take the values $1,2,3$ for the space coordinates $(r, \theta, \phi)$ which are spherical polar coordinates co-moving with the dust, and Greek indices take the values $(1,2,3,4)$ for the spacetime coordinates $(r, \theta, \phi, t)$. Semicolons and commas indicate covariant and partial derivatives respec- 
tively, and the suffixes $r$ and $\theta$ denote partial differentiation with respect to $r$ and $\theta$. All the functions are assumed to depend on $r$ and $\theta$ only, or they are constant.

The results to be used in this work may be found in a number of different publications [2,3] but we shall use [2] where all the necessary equations have been collected together and written in terms of the cylindrical polar coordinates and time $(z, \rho, \phi, t)$. We shall transform those equations in [2] that are required here, to the spherical polar coordinates and time $(r, \theta, \phi, t)$ with $r=\left(\rho^{2}+z^{2}\right)^{1 / 2}, \tan \theta=\rho / z, \phi=\phi, \quad z=r \cos \theta$, $\rho=r \sin \theta$ and $t=t$.

The contravariant and covariant forms $u^{\lambda}$ and $u_{\lambda}$ of the 4-velocity are

$$
u^{\lambda}=\delta_{4}^{\lambda} F^{-1 / 2} \quad u_{\lambda}=F^{1 / 2}\left(-w \delta_{\lambda}^{3}+\delta_{\lambda}^{4}\right) .
$$

The electric 4-current $J^{\lambda}$, the electromagnetic 4-potential $A_{\lambda}$ and the Faraday tensor $F_{\lambda \mu}$, are

$$
\begin{aligned}
& J^{\lambda}=\sigma u^{\lambda} \\
& A_{\lambda}=A_{3} \delta_{\lambda}^{3}+A_{4} \delta_{\lambda}^{4} \\
& F_{\lambda \mu}=A_{\lambda, \mu}-A_{\mu, \lambda} .
\end{aligned}
$$

where $\sigma$ is the electric charge density. The EinsteinMaxwell field equations for charged dust are

$$
\begin{aligned}
& G_{\mu}^{\lambda}=-8 \pi T_{\mu}^{\lambda} \\
& F_{\lambda \mu, v}+F_{\mu v, \lambda}+F_{v \lambda, \mu}=0 \\
& \frac{1}{\sqrt{-g}}\left(\sqrt{-g} F^{\lambda v}\right)_{, v}=4 \pi J^{\lambda} .
\end{aligned}
$$

Here, $G_{\mu}^{\lambda}$ is the Einstein tensor

$$
G_{\mu}^{\lambda}:=R_{\mu}^{\lambda}-\frac{1}{2} \delta_{\mu}^{\lambda} R
$$

where $R_{\mu}^{\lambda}$ is the Ricci tensor of the spacetime defined by its fully covariant form as

$$
R_{\mu}^{\lambda}:=\Gamma_{\lambda \beta, \mu}^{\beta}-\Gamma_{\lambda \mu, \beta}^{\beta}+\Gamma_{\lambda \beta}^{\gamma} \Gamma_{\mu \gamma}^{\beta}-\Gamma_{\lambda \mu}^{\beta} \Gamma_{\beta \gamma}^{\gamma}
$$

with $\Gamma_{\lambda \mu}^{v}$ the Christoffel symbols of the second kind based on the metric of $V$ in Equation (1), $R=g^{\beta \gamma} R_{\beta \gamma}$ is the spacetime scalar curvature invariant and $g$ is the determinant of $g_{\lambda \mu}$. The total stress-energy tensor $T_{\mu}^{\lambda}$ is

$$
T_{\mu}^{\lambda}=M_{\mu}^{\lambda}+E_{\mu}^{\lambda}
$$

where

$$
\begin{gathered}
M_{\mu}^{\lambda}=\rho u^{\lambda} u_{\mu} \\
E_{\mu}^{\lambda}=\frac{1}{4 \pi}\left(\frac{1}{4} \delta_{\mu}^{\lambda} F^{\beta \gamma} F_{\beta \gamma}-F^{\lambda \beta} F_{\mu \beta}\right)
\end{gathered}
$$

are, respectively, the matter and electromagnetic stressenergy tensors and $\rho$ is the mass density.
Instead of expressing the electromagnetic field equations in 4-dimensional form as in Equations (5), we shall use the Maxwell form (Maxwell's equations), because we can make direct comparisons with the results from classical electromagnetic theory. The electric and magnetic intensities and corresponding inductions in 3-vector form, are $[4,5]$

$$
\begin{aligned}
& \boldsymbol{E}=E_{a}=F_{a 4} \quad \boldsymbol{D}=D^{a}=-\sqrt{F} F^{a 4}, \\
& \boldsymbol{H}=H_{a}=\frac{1}{2} \sqrt{F} e_{a k p} F^{k p} \quad \boldsymbol{B}=B^{a}=\frac{1}{2} e^{a k p} F_{k p}
\end{aligned}
$$

where $e_{a k p}=\sqrt{-\gamma} \varepsilon_{a k p}, \quad e^{a k p}=\varepsilon_{a k p} / \sqrt{-\gamma}$ are the completely antisymmetric permutation tensors, $\sqrt{-\gamma}=\sqrt{-g} / \sqrt{F}, \quad \gamma$ being the determinant of the spatial metric tensor $\gamma_{a b}$ which is given by

$\gamma_{a b}=g_{a b}+\gamma_{a} \gamma_{b}$ with $\gamma_{a}=g_{a 4} / \sqrt{F}$, and $\varepsilon_{a k p}$ is the Levi-Civita symbol. It is easy to show that $\sqrt{-\gamma}=F^{-3 / 2} r^{2} \sin \theta$.

The transformed equations (2.14) and (2.13) of [2] may be written as

$$
\begin{aligned}
& 4 \pi r^{2} \sin ^{2} \theta e^{\mu} J^{3}=K \nabla^{* 2} A_{4}+F \nabla^{* 2} A_{3} \\
& +K_{r} A_{4 r}+r^{-2} K_{\theta} A_{4 \theta}+F_{r} A_{3 r}+r^{-2} F_{\theta} A_{3 \theta} \\
& 4 \pi r^{2} \sin ^{2} \theta e^{\mu} J^{4}=-L \nabla^{* 2} A_{4}+K \nabla^{* 2} A_{3} \\
& -L_{r} A_{4 r}-r^{-2} L_{\theta} A_{4 \theta}+K_{r} A_{3 r}+r^{-2} K_{\theta} A_{3 \theta}
\end{aligned}
$$

where the operators $\nabla^{2}$ and $\nabla^{* 2}$ are defined by

$$
\begin{gathered}
\nabla^{2} \equiv \frac{\partial^{2}}{\partial r^{2}}+\frac{1}{r^{2}} \frac{\partial^{2}}{\partial \theta^{2}}+\frac{2}{r} \frac{\partial}{\partial r}+\frac{\cot \theta}{r^{2}} \frac{\partial}{\partial \theta} \\
\nabla^{* 2} \equiv \frac{\partial^{2}}{\partial r^{2}}+\frac{1}{r^{2}} \frac{\partial^{2}}{\partial \theta^{2}}-\frac{\cot \theta}{r^{2}} \frac{\partial}{\partial \theta} .
\end{gathered}
$$

Equations (12) and (13) are the detailed form of the source-containing Maxwell equations given in the second of (5).

The non-zero components of the Ricci tensor obtained from the transformed Equations (2.16)-(2.21) of [2] are:

$$
\begin{aligned}
R_{3}^{3}= & -R_{4}^{4}=\frac{1}{2} e^{-\mu}\left\{\frac{\nabla^{2} F}{F}-\frac{1}{F^{2}}\left(F_{r}^{2}+\frac{F_{\theta}^{2}}{r^{2}}\right)\right. \\
& +\frac{F^{2}}{r^{2} \sin ^{2} \theta}\left(w \nabla^{* 2} w+w_{r}^{2}+\frac{w_{\theta}^{2}}{r^{2}}\right) \\
& \left.+\frac{2 F w}{r^{2} \sin ^{2} \theta}\left(F_{r} w_{r}+\frac{F_{\theta} w_{\theta}}{r^{2}}\right)\right\} \\
R_{1}^{1}= & -\frac{1}{2} e^{-\mu}\left\{\mu_{r r}+\frac{\mu_{\theta \theta}}{r^{2}}+\frac{\mu_{\theta} \cot \theta}{r^{2}}\right. \\
& \left.+\frac{F_{r}^{2}}{F^{2}}-\frac{2 F_{r}}{r F}-\frac{F^{2} w_{r}^{2}}{r^{2} \sin ^{2} \theta}\right\}
\end{aligned}
$$




$$
\begin{aligned}
& R_{2}^{2}=-\frac{1}{2} e^{-\mu}\left\{\mu_{r r}+\frac{2 \mu_{r}}{r}+\frac{\mu_{\theta \theta}}{r^{2}}-\frac{\mu_{\theta} \cot \theta}{r^{2}}\right. \\
&\left.+\frac{F_{\theta}^{2}}{r^{2} F^{2}}-\frac{2 F_{\theta} \cot \theta}{r^{2} F}-\frac{F^{2} w_{\theta}^{2}}{r^{4} \sin ^{2} \theta}\right\} \\
& R_{4}^{3}=-\frac{e^{-\mu}}{2 r^{2} \sin ^{2} \theta}\left\{F \nabla^{* 2} w+2 F\left(F_{r} w_{r}+\frac{F_{\theta} w_{\theta}}{r^{2}}\right)\right\} \\
& R_{3}^{4}= \frac{2 K R_{3}^{3}}{F}-\frac{\left(r^{2} \sin ^{2} \theta-K^{2}\right) R_{4}^{3}}{F^{2}} \\
& R_{1}^{2}= \frac{R_{2}^{1}}{r^{2}}=\frac{e^{-\mu}}{2 r^{2}}\left\{-\frac{F_{r} F_{\vartheta}}{F^{2}}\right. \\
&+\frac{1}{F \sin \theta}\left(F_{r} \cos \theta+\frac{F_{\theta} \sin \theta}{r}\right) \\
&\left.+\frac{1}{\sin \theta}\left(\mu_{r} \cos \theta+\frac{\mu_{\theta} \sin \theta}{r}\right)+\frac{F^{2} w_{r} w_{\theta}}{r^{2} \sin ^{2} \theta}\right\} \\
& R=-e^{-\mu}\left\{\mu_{r r}+\frac{\mu_{\theta \theta}}{r^{2}}+\frac{\mu_{r}}{r}+\frac{1}{2 F^{2}}\left(F_{r}^{2}+\frac{F_{\theta}^{2}}{r^{2}}\right)\right. \\
&\left.-\frac{1}{r F}\left(F_{r}+\frac{F_{\theta} \cot \theta}{r}\right)-\frac{F^{2}}{2 r^{2} \sin ^{2} \theta}\left(w_{r}^{2}+\frac{w_{\theta}^{2}}{r^{2}}\right)\right\}
\end{aligned}
$$

The entire Riemannian spacetime $V$, will be separated into the following 4-dimensional manifolds: the hypersurface $\Sigma$ with equation $r=a$ separates $V$ into the interior $V^{-}(0 \leq r \leq a)$ and exterior $V^{+}(a \leq r<\infty)$ spacetimes. We shall use the + and - signs to denote quantities in $V^{+}$and $V^{-}$whenever it is necessary to do so. Quantities without the + or - indicators, may be associated either with $V^{+}$or with $V^{-}$.

\section{The Exterior Solution}

In accordance with the formalism in [2], we first form the complex function

$$
\Phi=\eta+i \zeta
$$

where $\eta$ and $\zeta$ are harmonic functions. With a star denoting complex conjugation, the metric functions $F^{+}$ and $\mu^{+}$are then given by

$$
\left(F^{+}\right)^{-1}=\exp \left(\mu^{+}\right)=\Phi \Phi^{*}=\eta^{2}+\zeta^{2} .
$$

If we denote the real and imaginary parts of $\Phi^{-1}$ by $\varphi$ and $\psi$, then

$$
\varphi=\frac{\eta}{\eta^{2}+\zeta^{2}} \quad \psi=-\frac{\zeta}{\eta^{2}+\zeta^{2}} .
$$

We now choose the functions $\eta$ and $\zeta$ as follows:

$$
\begin{array}{ll}
\eta=b C(r) & C(r)=1+\frac{m}{r} \\
a \leq r<\infty & 0 \leq \theta \leq \pi
\end{array}
$$

$$
\zeta(r, \theta)=\left\{\begin{array}{l}
b\left\{-\lambda C(r)+\sum_{n=1}^{\infty} A_{2 n-1} a^{2 n} r^{-2 n} P_{2 n-1}(\cos \theta)\right\} \\
a \leq r<\infty \quad 0 \leq \theta<\pi / 2 \\
0 \\
a \leq r<\infty \quad \theta=\pi / 2 \\
b\left\{\begin{array}{l}
\left.+\lambda C(r)+\sum_{n=1}^{\infty} A_{2 n-1} a^{2 n} r^{-2 n} P_{2 n-1}(\cos \theta)\right\} \\
a \leq r<\infty
\end{array} \pi / 2<\theta \leq \pi\right.
\end{array}\right.
$$

where $b, m$ and $\lambda$ are constants whose significance will emerge later. From now on we shall omit writing the argument $\cos \theta$ of the Legendre polynomials and we shall write, for example, $P_{2 n-1}$ instead of $P_{2 n-1}(\cos \theta)$. We note the significant fact that at $\theta=\pi / 2, P_{2 n-1}=0$; this enables us to set $\zeta(r, \theta)=0$ at $\theta=\pi / 2 \quad a \leq r<\infty$ as in (27).

The function $w^{+}:=K^{+} / F^{+}$and the electromagnetic 4potential $A_{\lambda}^{+}=A_{3}^{+} \delta_{\lambda}^{3}+A_{4}^{+} \delta_{\lambda}^{4}$ in the exterior are obtained from

$$
\begin{aligned}
& w_{r}^{+}=2 \eta \zeta_{\theta} \sin \theta \\
& w_{\theta}^{+}=2 r^{2} \sin \theta\left(\zeta \eta_{r}-\eta \zeta_{r}\right) \\
& A_{3 r}^{+}=-w^{+} \varphi_{r}+\left(F^{+}\right)^{-1} \psi_{\theta} \sin \theta \\
& A_{3 \theta}^{+}=-w^{+} \varphi_{\theta}-\left(F^{+}\right)^{-1} \psi_{r} r^{2} \sin \theta \\
& A_{4}^{+}=-b+\varphi
\end{aligned}
$$

where an arbitrary constant in $A_{4}^{+}$was set equal to $b$ in order to satisfy the continuity condition of $A_{4}$. Note that the full expression for $w_{r}^{+}$in the first of (28) is $w_{r}^{+}=-2 \sin \theta\left(\zeta \eta_{\theta}-\eta \zeta_{\theta}\right)$, but by (26), $\eta_{\theta}=0$.

From Equations (24) and (28)-(31), we obtain the following expressions for $\left(F^{+}\right)^{-1}, \exp \left(\mu^{+}\right), w^{+}, A_{3}^{+}$ and $A_{4}^{+}$:

$$
\begin{aligned}
\left(F^{+}\right)^{-1}= & \exp \left(\mu^{+}\right)=b^{2} C^{2}(r) \\
& +b^{2}\left\{\mp \lambda C(r)+\sum_{n=1}^{\infty} A_{2 n-1} a^{2 n} r^{-2 n} P_{2 n-1}\right\}^{2} \\
w^{+} \equiv & \frac{K^{+}}{F^{+}}=2 b^{2} \sum_{n=1}^{\infty} \frac{A_{2 n-1}}{4 n-1} a^{2 n} \\
\times & \left\{2 n C(r) r^{1-2 n}-m r^{-2 n}\right\}\left(P_{2 n-2}-P_{2 n}\right) \\
A_{3}^{+}= & -b C(r) F^{+} w^{+} \\
+ & b^{2} \sum_{n=1}^{\infty} \frac{A_{2 n-1}}{4 n-1}\left\{2 n C(a) a^{2 n} r^{1-2 n}-m\right\} \\
\times & \left(P_{2 n-2}-P_{2 n}\right) \\
A_{4}^{+}= & b F^{+} C(r)-b .
\end{aligned}
$$


It is a little difficult to solve the two equations in (28) to find $w^{+}$in (33). It is even more difficult to solve the two Equations (29)-(30) to find $A_{3}^{+}$in (34) and complete details of the calculation are not given. Whenever there are two signs in a term, the upper sign gives the expression in $0 \leq \theta<\pi / 2$ and the lower sign the expression in $\pi / 2<\theta \leq \pi$, as in Equation (32).

The function $B$ defined by

$$
B=\left\{\begin{array}{cc}
+\lambda C(a) & 0 \leq \theta<\pi / 2 \\
0 & \theta=\pi / 2 \\
-\lambda C(a) & \pi / 2<\theta \leq \pi
\end{array}\right.
$$

has Legendre polynomial expansion of the form

$$
\begin{aligned}
& B=\sum_{n=1}^{\infty} A_{2 n-1} P_{2 n-1} \\
& A_{2 n-1}=\left(\frac{4 n-1}{2}\right) \int_{-1}^{1} B P_{2 n-1}(x) \mathrm{d} x
\end{aligned}
$$

where $A_{2 n-2}=0 n=1,2, \cdots$ It therefore follows from (27), that $\zeta(a, \theta)=0$. The function $B$ in (36) satisfies the conditions for such an expansion [6] and we have for the odd coefficients $A_{2 n-1}$

$$
\begin{aligned}
& A_{2 n-1}=\frac{(-1)^{n-1}(4 n-1)(2 n-2) ! n}{2^{2 n-1}(n !)^{2}} \lambda C(a), \\
& n=1,2, \cdots
\end{aligned}
$$

With $\zeta(a, \theta)=0$, by Equations (24) and (26), the metric function $F^{+}$at $r=a$ becomes $F^{+}(a, \theta)=1 / \eta^{2}=1 / b^{2} C^{2}(a)$. It will be shown in Section 4 , that $F^{-}(r, \theta)=1$ everywhere in the interior. In order to satisfy the junction condition at $r=a$ therefore, we must have $F^{+}(a, \theta)=1 / b^{2} C^{2}(a)=1$. It is easily seen that, as $r \rightarrow \infty, F(r, \theta) \rightarrow 1 / b^{2}\left(1+\lambda^{2}\right)$, which is a constant. If we take this to be equal to 1 , we obtain $b^{2}\left(1+\lambda^{2}\right)=1$ and collecting these relationships together we have

$$
\begin{aligned}
& b^{2}\left(1+\lambda^{2}\right)=1 \quad b^{2}=\frac{1}{C^{2}(a)} \\
& \lambda^{2}=\frac{2 m}{a}+\left(\frac{m}{a}\right)^{2} .
\end{aligned}
$$

The third of Equation (39) is the result of substituting the second of these equations into the first, bearing in mind the second of Equation (26) for $r=a$.

For the calculations that follow the functions $X$ and $Y$ defined by

$$
\begin{gathered}
X=\sum_{n=1}^{\infty} A_{2 n-1} 2 n a^{2 n} r^{-2 n-1} P_{2 n-1} \\
Y=\sum_{n=1}^{\infty} \frac{A_{2 n-1}}{4 n-1} 2 n(2 n-1) a^{2 n} r^{-2 n}\left(P_{2 n-2}-P_{2 n}\right)
\end{gathered}
$$

will be required. We express $\eta_{r}, \zeta_{r}$ and $\zeta_{\theta}$ as

$$
\eta_{r}=-\frac{b m}{r^{2}} \quad \zeta_{r}= \pm \frac{b \lambda m}{r^{2}}-b X \quad \zeta_{\theta}=-\frac{b Y}{\sin \theta} .
$$

The components of $G_{\mu}^{\lambda}$ are therefore calculated using the exterior functions (32)-(35) with Equations (16)-(22) and, whenever necessary, bearing in mind the first of Equation (39). The calculations give the following nonzero components:

$$
\begin{aligned}
{ }^{+} G_{1}^{1}= & -\left({ }^{+} G_{2}^{2}\right)=-8 \pi^{+} E_{1}^{1} \\
= & \left(F^{+}\right)^{2}\left\{-\frac{m^{2}}{r^{4}} \pm \frac{2 b^{2} \lambda m X}{r^{2}}-b^{2} X^{2}+\frac{b^{2} Y^{2}}{r^{2} \sin ^{2} \theta}\right\} \\
{ }^{+} G_{2}^{1}= & r^{2}\left({ }^{+} G_{1}^{2}\right)=-8 \pi^{+} E_{2}^{1} \\
= & 2 b^{2}\left(F^{+}\right)^{2}\left( \pm \frac{\lambda m}{r^{2}}-X\right) \frac{Y}{\sin \theta} \\
{ }^{+} G_{4}^{3}= & -8 \pi^{+} E_{4}^{3}=\frac{2 b^{2} m\left(F^{+}\right)^{3} Y}{r^{4} \sin ^{2} \theta} \\
{ }^{+} G_{3}^{4}= & -8 \pi^{+} E_{3}^{4} \\
= & 2\left\{\left(F^{+}\right)^{2} w^{+}\left(\frac{m^{2}}{r^{4}} \mp \frac{2 b^{2} \lambda m X}{r^{2}}+b^{2} X\right)\right. \\
& -\frac{b^{2} F^{+} m Y}{r^{2}}+\frac{b^{2}\left(F^{+}\right)^{2} w^{+} Y^{2}}{r^{2} \sin ^{2} \theta} \\
& \left.-\frac{b^{2}\left(F^{+}\right)^{3}\left(w^{+}\right)^{2} m Y}{r^{4} \sin ^{2} \theta}\right\} \\
& \left.+b^{2} X^{2}+\frac{b^{2} Y^{2}}{r^{2} \sin ^{2} \theta}-\frac{b^{2}\left(F^{+}\right) m w^{+} Y}{r^{4} \sin ^{2} \theta}\right\} \\
{ }^{+} G_{4}^{4}= & -\left({ }^{+} G_{3}^{3}\right)=-8 \pi^{+} E_{4}^{4} \\
= & -\left(F^{+}\right)^{2}\left\{\frac{m^{2}}{r^{4} \mp} \frac{2 b^{2} \lambda m X}{r^{2}}\right.
\end{aligned}
$$

Here, ${ }^{+} E_{\mu}^{\lambda}$ are the nonzero components of the electromagnetic energy tensor. The components of ${ }^{+} E_{\mu}^{\lambda}$ were obtained from (10) the third of (3) for $F_{\lambda \mu}$, the exterior electromagnetic potentials in (34) and (35). Equation (22) gives $R=0$ in $V^{+}$and so by (6), $G_{\mu}^{\lambda}=R_{\mu}^{\lambda}$ whether $\lambda$ is equal to $\mu$ or not. Another consequence of the result $R=0$, is that the matter energy tensor $M_{\mu}^{\lambda}$ will be null as should be the case in the electrovac $V^{+}$.

The sourceless Maxwell equations in the first of (5) give $F_{41, \theta}^{+}-F_{42, r}^{+}=0$ and $F_{23, r}^{+}+F_{31, \theta}^{+}=0$. By the third of (3) and with $A_{3}^{+}$and $A_{4}^{+}$given by (34) and (35), these become $-A_{3, \theta r}^{+}+A_{3, r \theta}^{+}=0, A_{4, r \theta}^{+}-A_{4, \theta r}^{+}=0$ which 
are trivially satisfied. The source-containing Maxwell Equations (12) and (13) with $A_{3}^{+}$and $A_{4}^{+}$given by Equations (34) and (35) will give $J^{3}=0$ and $J^{4}=0$ and so the 4-current is null in the electrovac $V^{+}$.

\section{The Interior Solution}

In accordance with the results of [2], the functions $F^{-}, \mu^{-}$and $A_{4}^{-}$are constant which we shall take as

$$
F^{-}=1 \quad \mu^{-}=0 \quad A_{4}^{-}=1-b
$$

The functions $K^{-}$and $A_{3}^{-}$satisfy an equation of the form $\nabla^{* 2} \Psi=0$ with $\nabla^{* 2}$ given by (15). This implies that $K^{-}$for example, is obtained from

$$
K^{-}=r \sin \theta\left(\xi_{r} \sin \theta+r^{-1} \xi_{\theta} \cos \theta\right)
$$

where $\xi$ is a harmonic function, which therefore satisfies Laplace's equation $\nabla^{2} \xi=0$ with $\nabla^{2}$ given by (14).

We choose $\xi$ as

$$
\xi= \pm \sum_{n=1}^{\infty} D_{2 n-1} r^{2 n} P_{2 n}
$$

where the constants $D_{2 n-1}$ are determined from the junction condition $K^{-}(a, \theta)=K^{+}(a, \theta)$. We use Equation (49) for $K^{-}$with $\xi$ given by (50) to find

$$
\begin{aligned}
K^{-}= & \sum_{n=1}^{\infty} D_{2 n-1} 2 n r^{2 n}\left[\left(1-\cos ^{2} \theta\right) P_{2 n}\right. \\
& \left.+\frac{2 n+1}{4 n+1}\left(P_{2 n+1}-P_{2 n-1}\right) \cos \theta\right]
\end{aligned}
$$

We can further show that

$$
\begin{aligned}
& \left(1-\cos ^{2} \theta\right) P_{2 n} \\
& =P_{2 n}-\frac{\cos \theta}{4 n+1}\left(2 n P_{2 n-1}+(2 n+1) P_{2 n+1}\right)
\end{aligned}
$$

and with this, the above expression for $K^{-}$becomes

$$
K^{-}=\sum_{n=1}^{\infty} D_{2 n-1} 2 n r^{2 n}\left(P_{2 n}-\cos \theta P_{2 n-1}\right) .
$$

Finally, after a little manipulation, the above expression for $K^{-}$becomes

$$
K^{-}=-\sum_{n=1}^{\infty} D_{2 n-1} \frac{2 n(2 n-1)}{4 n-1} \times r^{2 n}\left(P_{2 n-2}-P_{2 n}\right) .
$$

The junction condition for the continuity of $K$ implies that on $r=a, K^{-}(a, \theta)=K^{+}(a, \theta)$. Using the expression (33) for $w^{+}$and bearing in mind that $F^{+}(a, \theta)=1$, we have $K^{+}(a, \theta)=w^{+}(a, \theta)$. It therefore follows from (33) and (51), that the constants $D_{2 n-1}$ are given by

$$
-D_{2 n-1} 2 n(2 n-1) a^{2 n}=2 b^{2} A_{2 n-1}(2 n a C(a)-m) .
$$

This implies that $K^{-}$, but also $w^{-}$and $A_{3}^{-}$are given by

$$
\begin{aligned}
K^{-}= & w^{-}=-2 A_{3}^{-}=2 b^{2} \sum_{n=1}^{\infty} \frac{A_{2 n-1}}{4 n-1} a^{-2 n} r^{2 n} \\
& \times\{2 n a C(a)-m\}\left(P_{2 n-2}-P_{2 n}\right) .
\end{aligned}
$$

The functions $Z$ and $U$ defined by

$$
\begin{aligned}
Z & =\sum_{n=1}^{\infty} \frac{A_{2 n-1}}{4 n-1}(2 n a C(a)-m) \\
& \times 2 n a^{-2 n} r^{2 n-1}\left(P_{2 n-2}-P_{2 n}\right) \\
U & =\sum_{n=1}^{\infty} A_{2 n-1}(2 n a C(a)-m) a^{-2 n} r^{2 n} P_{2 n-1}
\end{aligned}
$$

will be required to simplify the components of the Einstein tensor.

The components of ${ }^{-} G_{\mu}^{\lambda}$ are calculated using the interior functions (48) and (52) with Equations (16)-(22) and, whenever necessary, bearing in mind the first of Equation (39). The calculations give the following nonzero components:

$$
\begin{aligned}
& -G_{1}^{1}=-\left({ }^{-} G_{2}^{2}\right)=-8 \pi^{-} E_{1}^{1}=-b^{4}\left(\frac{U^{2}}{r^{4}}-\frac{Z^{2}}{r^{2} \sin ^{2} \theta}\right) \\
& { }^{-} G_{3}^{3}=-8 \pi^{-} E_{3}^{3}=b^{4}\left(\frac{U^{2}}{r^{4}}+\frac{Z^{2}}{r^{2} \sin ^{2} \theta}\right) \\
& { }^{-} G_{4}^{4}=-8 \pi\left({ }^{-} M_{4}^{4}+{ }^{-} E_{4}^{4}\right)=-3 b^{4}\left(\frac{U^{2}}{r^{4}}+\frac{Z^{2}}{r^{2} \sin ^{2} \theta}\right) \\
& { }^{-} G_{3}^{4}=-8 \pi\left({ }^{-} M_{3}^{4}+{ }^{-} E_{3}^{4}\right)=4 b^{4}\left(\frac{U^{2}}{r^{4}}+\frac{Z^{2}}{r^{2} \sin ^{2} \theta}\right) w^{-} \\
& { }^{-} G_{2}^{1}=r^{2}\left({ }^{-} G_{1}^{2}\right)=-8 \pi^{-} E_{2}^{1}=\frac{2 b^{4} U Z}{r^{2} \sin \theta} .
\end{aligned}
$$

Here, ${ }^{-} E_{\mu}^{\lambda}$ and ${ }^{-} M_{\mu}^{\lambda}$ are the nonzero components of the electromagnetic and mass energy tensors respectively. The components of ${ }^{-} E_{\mu}^{\lambda}$ were obtained from (10) the third of (3) for $F_{\lambda \mu}$, the interior functions in (48) and (52). The components of ${ }^{-} M_{\mu}^{\lambda}$ were obtained from Equations (3) and (9) together with the interior functions (48) and (52). Equations (55)-(59) state that Einstein's Field Equations are satisfied in $V^{-}$. The sourceless Maxwell equations in the first of (5) give

$F_{23, r}^{-}+F_{31, \theta}^{-}=0$. By the third of (3) and with $A_{3}^{-}$given in Equation (52), this becomes $-A_{3, \theta r}^{-}+A_{3, r \theta}^{-}=0$ which is trivially satisfied. The source-containing Maxwell Equations (12) and (13) with $A_{4}^{-}$and $A_{3}^{-}$as in (48) and (52) respectively, will give

$$
{ }^{-} J^{3}=0-{ }^{-} J^{4}=\sigma^{-}=-\frac{b^{4}}{2 \pi}\left(\frac{U^{2}}{r^{4}}+\frac{Z^{2}}{r^{2} \sin ^{2} \theta}\right) .
$$

It is easily seen that 


$$
8 \pi^{-} M_{4}^{4}=8 \pi \rho^{-}=2 b^{2}\left(\frac{U^{2}}{r^{4}}+\frac{Z^{2}}{r^{2} \sin ^{2} \theta}\right) .
$$

It follows from this and Equation (60) that $\sigma^{-}=-2 \rho^{-}$ or, in dimensional units, $\sigma^{-}=-2 \sqrt{G} \rho^{-}$.

If $N$ is any function in $V$, we write

$$
\begin{aligned}
& {[N]:=N^{+}(a, \theta)-N^{-}(a, \theta)} \\
& N^{+}(a, \theta):=\lim _{\varepsilon \rightarrow 0} N(a+\varepsilon, \theta) \\
& N^{-}(a, \theta):=\lim _{\varepsilon \rightarrow 0} N(a-\varepsilon, \theta)
\end{aligned}
$$

where the second and third of Equation (61), represent the values of $N$ on the $V^{+}$and $V^{-}$sides of $\Sigma$.

It follows from Equations (32)-(35), (48) and (52) that $\left[g_{\alpha \beta}\right]=0$ and $\left[A_{\lambda}\right]=0$. The functions $g_{\alpha \beta}$ and $A_{\lambda}$ are therefore continuous across $\Sigma$, but one degree of smoothness is lost because the first order partial $r$-derivatives of these functions are discontinuous on $\Sigma$. It follows that the ordinary junction conditions requiring the continuity of the directional derivatives of these functions normal to $\Sigma$, cannot be applied. The discontinueties of these normal derivatives will generate a surface layer on $\Sigma$ with surface stress-energy tensor and surface 4-current and a more complicated set of junction conditions will apply. The Equations (12) and (13) for $J^{3}$ and $J^{4}$ will give rise to expressions with factors of delta-functions and first order partial $r$-derivatives which are discontinuous on $\Sigma$. We shall denote these terms by Gothic symbols, and we find from (12) and (13) that these are

$$
\begin{aligned}
& 4 \pi r^{2} \sin ^{2} \theta e^{\mu} \mathfrak{J}^{3} \\
& =\left\{K A_{4 r}^{+}+F\left(A_{3 r}^{+}-A_{3 r}^{-}\right)\right\} \times \delta(r-a) \\
& 4 \pi r^{2} \sin ^{2} \theta e^{\mu} \mathfrak{J}^{4} \\
& =\left\{-L A_{4 r}^{+}+K\left(A_{3 r}^{+}-A_{3 r}^{-}\right)\right\} \times \delta(r-a)
\end{aligned}
$$

where $A_{3}^{+}, A_{4}^{+}$and $A_{3}^{-}$, are given in (34), (35) and (52) respectively. To obtain the surface 4-current $s$ and $s^{4}$, we form the integrals of $J^{3}$ and $J^{4}$ with respect to proper distance measured perpendicularly through $\Sigma$ from $r=a-\varepsilon$ to $r=a+\varepsilon$ and then find the limits as $\varepsilon \rightarrow 0$. There are no sign indicators with the metric functions $K, F$ and $L$ in (62) and (63) because their values in both, $V^{+}$and $V^{-}$, are required in these integrations, where the only nonzero contributions will arise from the delta-function parts $\mathfrak{J}^{3}$ and $\mathfrak{J}^{4}$ of $J^{3}$ and $J^{4}$ in Equations (62) and (63). With $\hat{\boldsymbol{\varphi}}$ the unit vector in the $\phi$ direction, this gives

$$
\boldsymbol{s}=s^{3} \hat{\boldsymbol{\varphi}}=\frac{b^{3}}{2 \pi a^{2} \sin ^{2} \theta}\{Y(a, \theta)+b Z(a, \theta)\} \hat{\boldsymbol{\varphi}}
$$

$$
\boldsymbol{s}^{4}=-\frac{b m}{4 \pi a^{2}}+\frac{b^{3}}{2 \pi a^{2} \sin ^{2} \theta} w(a, \theta)\{Y(a, \theta)+b Z(a, \theta)\}
$$

The electromagnetic junction conditions are

$$
\begin{aligned}
{[\boldsymbol{D} \cdot \hat{\boldsymbol{n}}] } & =4 \pi \boldsymbol{s}^{4} \\
& =-\frac{b m}{a^{2}}+\frac{2 \mathrm{~b}^{3}}{a^{2} \sin ^{2} \theta} w^{-}(a, \theta)\{Y(a, \theta)+b Z(a, \theta)\} \\
{[\boldsymbol{H}] } & =4 \pi \boldsymbol{s} \times \hat{\boldsymbol{n}}=\frac{2 b^{3}}{\sin \theta}\{Y(a, \theta)+b Z(a, \theta)\} \hat{\boldsymbol{\theta}}
\end{aligned}
$$

where $\hat{\boldsymbol{n}}$ is the unit normal to the sphere and $\hat{\boldsymbol{\theta}}$ is the unit vector in the $\theta$ direction. In these equations, the contravariant component $D^{1}$ of $\boldsymbol{D}$ and the covariant component $\mathrm{H}_{2}$ of $\boldsymbol{H}$ from the second and third of Equation (11) were used.

The Equations (16)-(22) for $R_{\mu}^{\lambda}$ and $R$, will give rise to terms with factors of delta-functions and first order partial $r$-derivatives which are discontinuous on $\Sigma$.

Denoting these terms by Gothic symbols, the Einstein tensor $\mathfrak{G}_{\mu}^{\lambda}$ and the associated matter stress-energy tensor $\mathfrak{M}_{\mu}^{\lambda}$ are connected through the field equations, and so on $\Sigma$ we have

$$
\mathfrak{G}_{\mu}^{\lambda}:=\mathfrak{R}_{\mu}^{\lambda}-\frac{1}{2} \delta_{\mu}^{\lambda} \mathfrak{R} \quad \mathfrak{G}_{\mu}^{\lambda}=-8 \pi \mathfrak{M}_{\mu}^{\lambda}
$$

Bearing in mind that $\exp (-\mu)=F$ and that in $V^{-}$, $\exp \left(-\mu^{-}\right)=F^{-}=1$, we display below the components $\mathfrak{R}_{1}^{1}$ and $\mathfrak{R}_{2}^{2}$ as examples:

$$
\mathfrak{R}_{1}^{1}=\mathfrak{R}_{2}^{2}=-\frac{1}{2} \delta(r-a) F \mu_{r}^{+} .
$$

The surface stress-energy tensor $S_{\mu}^{\lambda}$ is expressed in terms of the limits as $\varepsilon \rightarrow 0$ of the integrals of $\mathfrak{M}_{\mu}^{\lambda} e^{\mu / 2}$ with respect to $r$ from $r=a-\varepsilon$ to $r=a+\varepsilon$ and with $\mathfrak{M}_{\mu}^{\lambda}$ given in Equations (64). The junction conditions on $\Sigma$ are [2,7]

$$
\begin{aligned}
& -2 G_{1}^{1}={ }^{(3)} R+k_{a}^{b} k_{b}^{a}-k^{2} \\
& G_{2}^{1} n_{1}=k_{2 ; b}^{b}-k_{; 2} \\
& 8 \pi S_{a}^{b}=\left[k_{a}^{b}\right]-\delta_{a}^{b}[k] \\
& S_{2 ; b}^{b}+\left[T_{2}^{1}\right] n_{1}=0 .
\end{aligned}
$$

Here, $k_{a b}$ is the extrinsic curvature tensor of $\Sigma$ defined by $k_{a b}=-n_{a: b}$, where the covariant differentiation is connected with the metric of $V$. Since $\mu=0$ on $\Sigma$ this gives $k_{a b}=g_{a b, 1} / 2$.

The hypersurface scalar curvature invariant of $\Sigma$ is ${ }^{(3)} R:={ }^{(3)} R_{a b} g^{a b}$ where the Ricci tensor ${ }^{(3)} R_{a b}$ is given by

$$
{ }^{(3)} R_{a b}=\Gamma_{a d, b}^{d}-\Gamma_{a b, d}^{d}+\Gamma_{a d}^{n} \Gamma_{b n}^{d}-\Gamma_{a b}^{d} \Gamma_{d n}^{n},
$$

$\Gamma_{a b}^{d}$ being the Christoffel symbols of the second kind based on the metric of $\Sigma$. With these, all the elements in 
the junction conditions (65) may be calculated and these conditions may be shown to be valid.

\section{Mass, Charge, Angular Momentum and the Magnetic Dipole Moment}

The mass, charge and angular momentum are defined by their imprints on the spacetime geometry far from the source. To obtain the gravitational mass and electric charge therefore, we expand the exterior metric function $F^{+}$up to the term $3 m^{2} / r^{2}$. Bearing in mind the first of (39), we then obtain from (32)

$$
F^{+}=\frac{1}{C^{2}(r)}=\left(1+\frac{m}{r}\right)^{-2}=1-\frac{2 m}{r}+\frac{3 m^{2}}{r^{2}} .
$$

We may transform $F^{+}$in (66) to the $F_{R N}$ of the Reissner-Nordstrom solution, by the transformation $r=\bar{r}-m \quad$ giving $F_{R N}=1-\frac{2 m}{\bar{r}}+\frac{q^{2}}{\bar{r}^{2}} \quad$ with $\quad q= \pm m$ [8], or in physical units, $F_{R N}=1-\frac{2 G m}{c^{2} \bar{r}}+\frac{G^{2} m^{2}}{c^{4} \bar{r}^{2}}$ with $q= \pm \sqrt{G} m$. This expression therefore implies that the gravitational mass is $m$ and the electric charge is $q$ and these are connected by [8]

$$
q= \pm \sqrt{G} m
$$

If we now expand $K^{+}$to $\mathrm{O}(1 / r)$ we have

$$
K^{+}=\frac{2 b^{2} A_{1} a^{2} \sin ^{2} \theta}{r}
$$

where $A_{1}$ is obtained from (38) by setting $n=1$, which will then give, bearing in mind (39)

$$
A_{1}=\frac{3 \lambda C(a)}{2}=\frac{3 \lambda}{2 b} \text {. }
$$

If $J$ is the total angular momentum, we have [9]

$$
K^{+}=\frac{2 J \sin ^{2} \theta}{r}
$$

From (68) and (70), we then obtain $J=b^{2} a^{2} A_{1}$ and on using (69), this gives

$$
J=\frac{3}{2} b \lambda a^{2} .
$$

The dipole field is the part of the magnetic field $\boldsymbol{H}$ whose physical components $H_{r}$ and $H_{\theta}$ contain the factors $r^{-3} \cos \theta$ and $r^{-3} \sin \theta$ respectively. Since only the $r^{-3}$ power is required, we only need the $n=1$ mode of the third of the expressions in (11) for $\boldsymbol{H}$. We find that these components are

$$
\begin{aligned}
& H_{r}=\frac{2 b^{3} A_{1} a^{2}\left(1-\lambda^{2}\right) \cos \theta}{r^{3}} \\
& H_{\theta}=\frac{b^{3} A_{1} a^{2}\left(1-\lambda^{2}\right) \sin \theta}{r^{3}}
\end{aligned}
$$

With these, the magnetic dipole moment is therefore, $P=b^{3} A_{1} a^{2}\left(1-\lambda^{2}\right)$ and on using (69), this gives

$$
P=\frac{3}{2} b^{3} A_{1} a^{2}\left(1-\lambda^{2}\right)
$$

From (71) and (73), we deduce that the gyromagnetic ratio is

$$
\frac{P}{J}=b\left(1-\lambda^{2}\right)
$$

In physical units Equations (71), (73), (74) and the third of (39), become

$$
\begin{aligned}
& J=\frac{3}{2} b \lambda a^{2}\left(\frac{c^{3}}{G}\right) \\
& P=\frac{3}{2} b^{2} a^{2} \lambda\left(1-\lambda^{2}\right) \frac{c^{2}}{\sqrt{G}} \\
& \frac{P}{J}=b\left(1-\lambda^{2}\right) \frac{\sqrt{G}}{c} \\
& \lambda^{2}=2 \frac{G m}{a c^{2}}+\left(\frac{G m}{a c^{2}}\right)^{2}
\end{aligned}
$$

It may be shown that the units of $J$ and $P$ are $[J]=M L^{2} T^{-1}$ and $[P]=M^{1 / 2} L^{5 / 2} T^{-1} \quad$ respectively, which are the units of angular momentum and magnetic dipole moment. We also find from the second of (26) and the second of (39) that

$$
C(a)=1+\frac{G m}{a c^{2}} \quad b=\frac{1}{C(a)}
$$

We stress the fact that all the above formulae are for an electrically charged sphere whose mass $m$ and charge $q$ are related by Equation (67). We note from (75) and (76) that the angular momentum $J$ and dipole moment $P$ depend on $a^{2}$ but also in a somewhat more subtle way, on the mass to radius ratio through the quantities $\lambda$ and $b$. The analytical Formula (77) may be applied to a number of different objects. We note that there exists a formula for the gyromagnetic ratio of stars known as Blackett's empirical Formulas [10-12], which reads

$$
\frac{P}{J}=\beta \frac{\sqrt{G}}{c}
$$

where $\beta$ is a constant of the order of unity so that (80) becomes

$$
\frac{P}{J}=\left(\frac{\sqrt{G}}{c}\right) .
$$

Blackett suggested that an explanation of this relation "must be sought in a new fundamental property of matter not contained within the structure of present day physical theory." We note in this connection that the factor 
$\sqrt{G} / c$, occurs in both our analytical Formula (77) and in Blackett's empirical Formula (80). The explanation for the presence of this factor in the analytical Formula (77) however is implicit in its derivation. Furthermore, the coefficient of $\sqrt{G} / c$ in this formula is $b\left(1-\lambda^{2}\right)$, and in Blackett's Formula (80), it is a constant equal to 1, or approximately equal to 1 . The quantity $b\left(1-\lambda^{2}\right)$ with $\lambda$ and $b$ given by (78) and (79) respectively, is expected to vary from star to star, but $\beta$ in Blackett's Formula (80) is a constant equal to 1 for all stars, an assertion that seems improbable. In the context of our solution, it is difficult to see why different objects which can be as diverse as the Earth and the Sun, will conform to such a requirement as implied by Blackett's empirical Formula (81). Although the "new physics" idea was subsequently abandoned, it is nevertheless of interest to investigate further under what circumstances, if any, our exact analytical Formula (77) reduces to Blackett's empirical Formula (81).

In order to gain an insight into the relation between the analytical Formula (77) and Blackett's empirical Formula (81), we shall consider three cases with different numerical values for the radius $a$ and gravitational mass $m$ of the sphere. We shall then proceed to calculate the corresponding quantities in $\lambda^{2}, \lambda, b$ and $P / J$ in (78), (79) and (77):

$$
\begin{aligned}
& m=1.989 \times 10^{33} \mathrm{~g} \quad a=6.9599 \times 10^{10} \mathrm{~cm} \\
& \lambda^{2}=4.243406362 \times 10^{-6} \quad \lambda=2.059953 \times 10^{-3} \\
& b=0.999997878 \quad \frac{P}{J}=0.999993635 \frac{\sqrt{G}}{c} \\
& m=4.33602 \times 10^{33} \mathrm{~g} \quad a=1.4337394 \times 10^{11} \mathrm{~cm} \\
& \lambda^{2}=4.493 \times 10^{-6} \quad \lambda=2.119669786493 \times 10^{-3} \\
& b=0.999997755 \frac{P}{J}=0.999727877 \frac{\sqrt{G}}{c} \\
& m=5.976 \times 10^{27} \mathrm{~g} \quad a=6.3675 \times 10^{8} \mathrm{~cm} \\
& \lambda^{2}=1.393554681 \times 10^{-9} \quad \lambda=3.733034531 \times 10^{-5} \\
& b=0.999999999 \frac{P}{J}=0.999999998 \frac{\sqrt{G}}{c} .
\end{aligned}
$$

The above masses and radii were deliberately chosen to be numerically equal to those of the Sun, 78 Virginis and the Earth. These correspond to the three astronomical objects that are quoted in the literature by later authors in connection with Blackett's empirical Formula (80) [10]. It is seen from the numerical results in (82)-(84), that in the case of our electrically charged spheres, the coefficient of $\sqrt{G} / c$ is very nearly equal to 1 in every case. We must conclude that in situations where the ratio $m / a$ is such that $b\left(1-\lambda^{2}\right)$ is approximately equal to 1 , our analytical Formula (77) will give Blackett's empirical
Formula (81). These reductions however, are only possible in the cases where, $b\left(1-\lambda^{2}\right)=1$. Thus, if we consider a typical neutron star as a fourth case we have

$$
\begin{gathered}
m=1.4 M_{s}=2.7846 \times 10^{33} \mathrm{~g} \quad a=10^{6} \mathrm{~cm} \\
\lambda^{2}=0.4562170327 \quad \lambda=0.6754384003 \\
b=0.959011213 \quad \frac{P}{J}=0.521493962 \frac{\sqrt{G}}{c}
\end{gathered}
$$

where $M_{S}$ is the mass of the Sun.

It is seen that $P / J \neq \sqrt{G} / c$ and this is because $b\left(1-\lambda^{2}\right)=0.521493962 \neq 1$. In the context of our equations, we found the precise condition under which our analytical Formula (77) will give Blackett's empirical Formula (81). Again, in the context of our equations, this provides a full explanation why Blackett's formula is sometimes valid and why this occurs only for a range of objects. Our formula for the gyromagnetic ratio $P / J$ is not empirical, but an exact analytical formula which is a consequence of the equations derived from the exact global solution of the Einstein-Maxwell field equations found here. It does not require any new fundamental properties of matter or any new physics and it is valid for all values of the ratio $m / a$.

We note that Wilson $[12,13]$ observed that in the case of the Earth and the Sun, the Formula (80) can be accounted for, if we assume that a rotating mass $m$ has the same effect as a rotating electrical charge $q$ where $m$ and $q$ are connected by Equation (67). It is a little puzzling that our electrically charged spheres charged as they are in accordance with Equation (67), seem to echo the above observation by Wilson. In our case however, $m$ and $q$ are connected by Equation (67) in reality. The quantity of charge required is quite small. As noted by Bonnor [8], if the mass $m$ and charge $q$ are related by Equation (67), then if in a sphere of neutral hydrogen one atom in $10^{18}$ had lost its electron, this would be sufficient.

\section{Discussion and Conclusions}

Exact exterior and interior solutions of the EinsteinMaxwell field equations for rigidly rotating pressure-free matter were obtained. The exterior and interior spacetimes are separated by a boundary which is a surface layer with surface stress-energy tensor and surface electric 4-current.

Perhaps one of the most important aspects of this work is that the source of spacetime, is rotating charged matter bounded by a closed surface. As far as we know, a global solution with a volume distribution of finite bounded rotating matter as a source of the spacetime, does not exist in the literature, although flat disk solutions do indeed exist [1]. Another important outcome of this work is the derivation of analytical formulae for the angular mo- 
mentum, dipole moment and gyromagnetic ratio of a rotating sphere based on general relativistic equations.

The mass, charge, angular momentum and the magnetic dipole moment were determined in Section 5. In particular, we derived the analytical Formula (77) for the gyromagnetic ratio and discussed special cases to establish the facts regarding the connection between the analytical Formula (77) and Blackett's empirical for Formula (80) the conditions under which the analytical Formula (77) reduces to Blackett's empirical formula, were obtained. No new properties of matter and no new physics was required. Perhaps the analytical Formula (77) is valid for all rotating objects and in particular for stars, but we have no data to demonstrate this, except for the cases of the Sun, 78 Virginis and the Earth.

All the physical quantities of interest in the interior and exterior were calculated as well as those associated with the spherical surface layer. In this problem, the ordinary gravitational junction conditions are inappropriate. In fact there are two sets of junction conditions, the electromagnetic and the gravitational ones. The former were expressed in the familiar form of classical electromagnetic theory. The gravitational junction conditions in this problem are more complicated than the usual ones, because of the surface layer. These were clearly stated, although no detailed formulae were displayed.

This solution permits a reversal of the signs of $A_{3}^{+}$ and $A_{4}^{+}$in (34) and (35) [14], which will cause a reversal of the signs of $A_{3}^{-}$and $A_{4}^{-}$in (52) and (48). If we replace the harmonic functions $\eta$ and $\zeta$ in (26) and (27) by

$$
\eta=C(r)=1+\frac{m}{r} \quad \zeta=\frac{J \cos \theta}{r^{2}}
$$

then, instead of the metric functions in (32) and (33), we shall have

$$
\begin{gathered}
F^{+}=\left\{\left(1+\frac{m}{r}\right)^{2}+\left(\frac{J \cos \theta}{r^{2}}\right)^{2}\right\}^{-1} \\
w^{+}=\frac{J \sin ^{2} \theta}{r}\left(2+\frac{m}{r}\right)
\end{gathered}
$$

with appropriate modifications to the remaining functions in (32)-(34). Our exterior solution, given by these equations, reduces to the solution obtained by Perjes [14].

To find the limit of the exterior solution (32)-(35) when the angular momentum $J$ is reduced to zero, we replace the harmonic function $\zeta$ in (27) by zero, choose $b=1$, and base the solution on the single harmonic function $\eta=C(r)$ in (26). This leads to

$$
\begin{aligned}
& \left(F^{+}\right)^{-1}=\exp \left(\mu^{+}\right)=C^{2}(r) \quad K^{+}=w^{+}=0 \\
& A_{4}^{+}=\frac{1}{C(r)}-1 \quad A_{3}^{+}=0
\end{aligned}
$$

which is the Papapetrou solution [15] for which Bonnor has found a matching interior solution [8].

Referring to the surface layer that occurs in our solution, we note the result obtained by Ruffini and Treves in a non-relativistic treatment, in which they had shown that a magnetized rotating object has surface charge and current densities; it is also endowed with a net electric charge [16]. This agrees with our results and in particular, it confirms the existence of a surface layer with 4-current and stress-energy tensor on the boundary $r=a$.

The mass, charge, angular momentum and the magnetic dipole moment were determined in Section 5. In particular, we derived the analytical Formula (77) for the gyromagnetic ratio and discussed special cases to establish the facts regarding the connection between the analytical Formula (77) and Blackett's empirical Formula (80). The conditions under which the analytical Formula (77) reduces to Blackett's empirical formula, were obtained. No new properties of matter and no new physics were required. Perhaps the analytical Formula (77), is valid for all rotating objects and in particular for stars, but we have no data to demonstrate this, except for the cases of the Sun, 78 Virginis and the Earth.

\section{REFERENCES}

[1] M. A. H. MacCallum, M. Mars and P. Vera, "Stationary Axisymmetric Exteriors for Perturbations of Isolated Bodies in General Relativity, to Second Order," Physical Review D, Vol. 75, 2007, Article ID: 024017.

[2] A. Georgiou, "Rotating Einstein-Maxwell Fields: Smoothly Matched Exterior and Interior Spacetimes with Charged Dust and Surface Layers," Classical and Quantum Gravity, Vol. 11, No. 1, 1994, pp. 167-186. doi:10.1088/0264-9381/11/1/018

[3] W. Bonnor, "Rotating Charged Dust in General Relativity," Journal of Physics A: Mathematical and General, Vol. 13, 1980, pp. 3465-3477.

[4] A. Georgiou, "Comments on Maxwell's Equations," International Journal of Mathematical Education in Science and Technology, Vol. 13, No. 1, 1982, pp. 43-47. doi:10.180/0020739820130106

[5] C. Moller, "The Theory of Relativity," 2nd Edition, Oxford University Press, Oxford, 1972.

[6] E. T. Whittaker and G. N. Watson, "A Course of Modern Analysis," 4th Edition, Cambridge University Press, Cambridge, 1950.

[7] W. Israel, "Singular Hypersurfaces and Thin Shells in General Relativity," Nuovo Cimento B, Vol. 48, No. 2, 1966, p. 463. doi:10.1007/BF02712210

[8] W. Bonnor, "The Equilibrium of a Charged Sphere," Monthly Notices of the Royal Astronomical Society, Vol. 129, 1965, p. 433. http://adsabs.harvard.edu/abs/1965MNRAS.129.443B

[9] J. N. Islam, "Rotating Fields in General Relativity," Cambridge University Press, Cambridge, 1985. 


\section{doi:10.1017/CB09780511735738}

[10] P. M. S. Blackett, "The Magnetic Field of Massive Rotating Bodies," Nature, Vol. 159, No. 4046, 1947, pp. 658666.

[11] D. V. Ahluwalia and T. Y. Wu, "On the Magnetic Field of Cosmological Bodies," Lettere Al Nuovo Cimento, Vol. 23, No. 11, 1978, pp. 406-408. doi:10.1007/BF02786999

[12] J. A. Nieto, "Gravitational Magnetism and General Relativity," Revista Mexicana de Fisica, Vol. 34, No. 4, 1988, pp. 571-576.

[13] H. A. Wilson, "An Experiment on the Origin of the Earth's Magnetic Field," Proceedings of the Royal Soci- ety, Vol. 104A, 1923, p. 451.

http://www.jstor.org/stable/94216

[14] Z. Perjes, "Solutions of the Coupled Electromagnetic Equations Representing the Fields of Spinning Sources," Physical Review Letters, Vol. 27, 1971, p. 1668.

[15] A. Papapetrou, "A Static Solution of the Equations of the Gravitational Field for an Arbitrary Charge-Distribution," Proceedings of the Royal Irish Academy, Vol. 51, 19451948, p. 191.

[16] R. Ruffini and A. Treves, "On a Magnetized Rotating Sphere," Astrophysical Journal Letters, Vol. 13, 1973, p. 109. 